\title{
Endothelium injury and inflammatory state during abdominal aortic aneurysm surgery: scrutinizing the very early and minute injurious effects using endothelial markers - a pilot study
}

Michał Kokot ${ }^{1}$, Grzegorz Biolik², Damian Ziaja², Tadeusz Fojtㄹ, Kamila Cisak ${ }^{1}$, Katarzyna Antoniak ${ }^{1}$, Krzysztof Pawlicki ${ }^{3}$ Krzysztof Ziaja², Jan Duława ${ }^{1}$

1Department of Internal Medicine and Metabolic Diseases, Medical University of Silesia, Katowice, Poland

2Department of General and Vascular Surgery, Medical University of Silesia, Katowice, Poland

${ }^{3}$ Department of Biophysics, Medical University of Silesia, Katowice, Poland

Submitted: 24 April 2012

Accepted: 18 October 2012

Arch Med Sci 2013; 9, 3: 479-486

DOI: $10.5114 /$ aoms.2013.34412

Copyright $\odot 2013$ Termedia \& Banach

\begin{abstract}
Introduction: One of the most severe complications of repair surgery for abdominal aortic aneurysms (AAA) is acute kidney injury (AKI). Acute kidney injury is an inflammatory process whose pathogenesis involves endothelial cells (EC). The aim of this study was to assess the dynamics of endothelium injury markers measured during elective AAA surgery which might confirm the inflammatory character of AKI.

Material and methods: The study group consisted of 14 patients with AAA. We measured plasma soluble forms of intercellular adhesion molecule-1 (ICAM-1), vascular cell adhesion molecule-1 (VCAM-1), E-selectin, P-selectin as well as the levels of von Willebrand factor (VWF) before, during (including intra-abdominal vein levels before and after aortic clamp removal) and within 2 days after surgery.

Results: We have found a biphasic response of ICAM-1, VCAM-1 and P-selectin with an initial fall and subsequent rise. However, only VCAM-1 changes were significant compared to its baseline value. The maximum decrease of VCAM- 1 was observed in the renal vein 5 min after aortic clamp removal $(335.42 \pm 129.63 \mathrm{ng} / \mathrm{ml}$ vs. $488.90 \pm 169.80 \mathrm{ng} / \mathrm{ml}$ baseline value, $p<0.05)$, and the highest rise $48 \mathrm{~h}$ after aortic clamp removal (721.46 \pm 333.99 vs. baseline, $p<0.05)$.

Conclusions: Vascular cell adhesion molecule-1 turned out to be the most sensitive indicator of EC injury and inflammatory status after AAA surgery. During AAA surgery, soluble forms of P-selectin, ICAM-1 and VCAM-1 demonstrate a biphasic response with an initial fall and subsequent rise. These soluble forms could have a modulatory effect on the development of inflammation.
\end{abstract}

Key words: acute kidney injury, intercellular adhesion molecule-1, vascular cell adhesion molecule-1, E-selectin, P-selectin, von Willebrand factor.

\section{Introduction}

Acute kidney failure (AKF) and its milder form, acute kidney injury (AKI), is an ominous complication of a number of vascular surgery interventions. Even small increases in serum creatinine have been found to be associated with increased mortality $[1,2]$. The prevalence of AKI or overt AKF

\author{
Corresponding author: \\ Michat Kokot MD \\ Department \\ of Internal Medicine \\ and Metabolic Diseases \\ Medical University of Silesia \\ SPSK Nr 7 GCM \\ 47 Ziolowa St \\ 40-635 Katowice, Poland \\ Phone/fax: +48 322523593 \\ E-mail: metabol@gcm.pl
}


after cardiac surgery ranges from $1 \%$ to $30 \%$ of the patients, $1 \%$ of whom undergo dialysis, with mortality reaching $60-70 \%[3,4]$. Until now, investigations have mainly focused on detailed analysis of AKI after cardiac surgery [3]. Another frequent major vascular intervention, the operation of abdominal aortic aneurysm (AAA), is also associated with relatively common occurrence of AKI [5]. Bearing this in mind, the relationship between AAA surgery and AKI certainly deserves more attention. Abdominal aortic aneurysm surgery allows precise identification of the starting point and overall duration of factors resulting in renal injury. Hence, this intervention is a valuable research model of AKI.

The damage to renal tubular epithelium appears to be a key element in the pathogenesis of AKI. However, epithelial regeneration during recovery from AKI is rapid and usually complete. As opposed to tubular epithelium, the endothelium of intrarenal blood vessels has poor regenerative capabilities, which substantially contributes to the development of chronic kidney disease [6].

It has been widely acknowledged that the inflammatory process constitutes one of the most important mechanisms of AKI initiation and progression [7, 8]. Abdominal aortic aneurysm surgery is regarded as a trigger of potent inflammatory responses $[9,10]$.

Endothelial cells play a pivotal role during inflammatory processes both as their active participants and victims [11]. Acute kidney injury contributes to the injury of intra- and extrarenal endothelium. Pathological processes occurring in the endothelium of intrarenal blood vessels are important constituents of AKI pathogenesis [12]. Von Willebrand factor (VWF) and cell adhesion molecules (CAMs), i.e., intercellular adhesion molecule-1 (ICAM-1), vascular cell adhesion molecule-1 (VCAM-1), E-selectin and P-selectin, are important indicators of endothelial cell (EC) function and injury [9, 13-15].

Up till now, no efficacious preventive AKI therapy has been available except appropriate preoperative fluid supplementation [16]. Since the "window of effective therapeutic opportunity" is of relatively short duration (about 48-72 h) and should be placed in early-stage AKI, timely detection of this complication is of uppermost importance [17].

In the light of the above-mentioned facts we considered it interesting to try to elucidate the relationship between AAA surgery, AKI and endothelial injury.

The paper presents the preliminary results of a pilot study of a larger, ongoing project. The aim of this study was to scrutinize very early changes which might possibly occur in the levels of endothelium injury indicators; the occurrence of such changes would confirm the inflammatory aspect of
AKI triggered by AAA surgery. We hope that our investigations might shed more light on the complex interrelation between AKI and endothelial injury.

\section{Material and methods}

\section{Subjects}

The study comprised 14 consecutive patients admitted to the Department of Vascular Surgery within the period from February 2010 to September 2010 for elective surgical management of AAA, who signed informed consent and did not meet exclusion criteria including: (1) the use of aminoglycoside antibiotics within 1 month before surgery, (2) treatment with cyclosporine A, (3) neoplastic disease, (4) another surgical procedure during 1 month prior to enrollment, (5) stroke during the preceding 2 months, (6) myocardial infarction during the preceding 3 months, (7) essential psychiatric, metabolic, neurological, blood or major internal organ disorders, (8) incident of acute renal failure (ARF) or renal replacement therapy during the preceding 6 months, (9) an ongoing acute inflammatory response, (10) urinary tract obstruction.

The research was approved by the Bioethics Committee of the Medical University of Silesia. Patients' characteristics, comorbidities and basic laboratory data are shown in Table I. Before enrollment 4 patients were treated with statins, 6 with ACE-I, one with an AT1-blocker, 2 with nonsteroid anti-inflammatory drugs, 8 with $\beta$-blockers, 3 with Ca-channel blockers, 2 with nitrates, one with diuretics, but none with spironolactone and pentoxifylline. No patients received nephrotoxic drugs in the 2 weeks before or within the study period.

\section{Abdominal aortic aneurysm surgery}

Between days 2 and 108 before surgery the subjects underwent contrast-enhanced computed tomographic angiography. Following infrarenal cross-clamping, the aortic aneurysm was excised and the aorta was reconstructed using a PTFE graft. Surgery was performed under general anesthesia; details are given in Table II.

\section{Design}

The protocol of the study was as follows. All eligible patients underwent AAA and kidney ultrasound as well as renal artery Doppler examination. Blood samples were obtained (a) before surgery (day -1) from an upper extremity vein; (b) during surgery (day 0) from the accessible (left) renal vein prior to aortic clamping; (c) from the renal vein, (d) from the vena cava inferior, and (e) from an upper extremity vein ("c", "d" and "e") just before removal of the aortic clamp; and (f) from the renal vein and 
(g) from an upper extremity vein ("f" and "g") 5 min after removal of the aortic clamp. The successive blood samples were drawn from an upper extremity vein: (h) 2 h (day 0), (i) 4 h (day 0), (j) 12 h (day 0), (k) 24 h (day 1), (l) 48 h (day 2), (m) 72 h (day 3), (n) $96 \mathrm{~h} \mathrm{(day} \mathrm{4)} \mathrm{and} \mathrm{(o)} 120 \mathrm{~h}$ (day 5) following aortic clamp removal. The duration of surgery and aortic clamping, total volume of intravenous infusions and blood loss during surgery were all considered. Complete blood count ( $4 \mathrm{~h}$ after termination of surgery) and C-reactive protein (CRP) (72 h after surgery) were also assessed. Based on the literature on the subject, we chose the following serum or plasma indicators of inflammation and endothelium functions: ICAM-1, VCAM-1, P-selectin, E-selectin and von Willebrand factor antigen (vWF:Ag). Concentrations thereof in all (a-l) blood samples as well as creatinine concentrations in a, k, l, m, n, and o samples were determined. Estimated glomerular filtration rate (eGFR) was calculated using the Modification of Diet in Renal Disease Study (MDRD) equation and by the CKD-EPI Study formula [18, 19].

\section{Laboratory methods}

After collection and clotting for ICAM-1, VCAM-1, P-selectin, and E-selectin, blood samples were centrifuged for $15 \mathrm{~min}$ at $3000 \mathrm{~g}$ (rotation $4000 \cdot \mathrm{min}^{-1}$ ). Frozen serum or citrate-anticoagulated plasma was stored at $-80^{\circ} \mathrm{C}$. Serum creatinine concentrations were determined using Jaffe's colorimetric method. Serum ICAM-1, VCAM-1, P-selectin, E-selectin and plasma VWF:Ag concentrations were assessed using commercially available enzyme-linked immunosorbent assays (Quantikine Human sICAM-1/CD54 ELISA Kit; Quantikine Human sVCAM-1 ELISA Kit; Human soluble P-Selectin/CD62P ELISA Kit; Quantikine Human sE-Selectin/CD62E ELISA Kit, all manufactured by R\&D Systems, Inc., Minneapolis, United States of America; Von Willebrand Factor Antigen ELISA Kit manufactured by Helena BioSciences Europe) according to the manufacturer's instructions. Patient vWF:Ag in relative percent concentration was determined against a curve made from the reference plasma provided with the kit. Other laboratory parameters were determined using routine tests.

\section{Statistical analysis}

Data were analyzed using Statistica 8.0. computer software. All variables were tested for normality of distribution by means of the KolmogorovSmirnov test. Kruskal-Wallis/Mann-Whitney U and ANOVA tests were used for non-parametric and parametric analyses, respectively. The correlation rate was calculated using Spearman's test. The Spearman rank correlation coefficient (rs) was determined. All results were expressed as mean
Table I. Patients' characteristics

\begin{tabular}{|c|c|}
\hline \multicolumn{2}{|l|}{ Demographic parameters } \\
\hline Number of patients (males/females) & $14(12 / 2)$ \\
\hline Age [years] & $66.39 \pm 6.31$ \\
\hline Body mass index $\left[\mathrm{kg} / \mathrm{m}^{2}\right]$ & $26.41 \pm 3.86$ \\
\hline \multicolumn{2}{|l|}{ Comorbidities (number of patients) } \\
\hline Current smokers & 7 \\
\hline Diabetes & 3 \\
\hline Coronary artery disease & 5 \\
\hline Hypertension & 8 \\
\hline Peripheral vascular disease & 6 \\
\hline \multicolumn{2}{|l|}{ Aneurysm characteristics } \\
\hline Neck diameter $[\mathrm{mm}]$ & $24.43 \pm 6.54$ \\
\hline Neck length [mm] & $25.67 \pm 8.02$ \\
\hline Aneurysm diameter [mm] & $59.21 \pm 10.97$ \\
\hline \multicolumn{2}{|l|}{ Kidney long axis } \\
\hline Right kidney [mm] & $93.50 \pm 9.30$ \\
\hline Left kidney [mm] & $96.79 \pm 8.59$ \\
\hline \multicolumn{2}{|l|}{ Kidney Doppler examination } \\
\hline Right kidney pulsation index & $0.90 \pm 0.17$ \\
\hline Left kidney pulsation index & $0.88 \pm 0.21$ \\
\hline Right kidney resistance index & $0.61 \pm 0.08$ \\
\hline Left kidney resistance index & $0.60 \pm 0.10$ \\
\hline \multicolumn{2}{|l|}{ Blood count parameters } \\
\hline \multicolumn{2}{|c|}{ Erythrocyte count before surgery $\left[\times 10^{12} / I\right] 4.84 \pm 0.46$} \\
\hline \multicolumn{2}{|c|}{ Erythrocyte count $4 \mathrm{~h}$ after surgery $\left[\times 10^{12} / l\right] 3.91 \pm 0.48$} \\
\hline \multicolumn{2}{|c|}{$\begin{array}{l}\text { Hemoglobin concentration before surgery } 14.67 \pm 1.30 \\
{[\mathrm{mmol} / \mathrm{l}]}\end{array}$} \\
\hline \multicolumn{2}{|c|}{$\begin{array}{l}\text { Hemoglobin concentration } 4 \mathrm{~h} \text { after surgery } 11.69 \pm 1.45 \\
{[\mathrm{mmol} / \mathrm{l}]}\end{array}$} \\
\hline Hematocrit before surgery [\%] & $43.66 \pm 3.57$ \\
\hline Hematocrit $4 \mathrm{~h}$ after surgery [\%] & $35.48 \pm 4.15$ \\
\hline Platelet count before surgery $\left[\times 10^{9} / \mathrm{I}\right]$ & $228.07 \pm 57.15$ \\
\hline \multicolumn{2}{|c|}{ Platelet count $4 \mathrm{~h}$ after surgery $\left[\times 10^{9} / \mathrm{l}\right] 185.43 \pm 50.55^{\star}$} \\
\hline P-LCR before surgery [\%] & $28.91 \pm 7.21$ \\
\hline P-LCR $4 \mathrm{~h}$ after surgery [\%] & $27.79 \pm 6.77$ \\
\hline MPV before surgery [fl] & $10.50 \pm 0.98$ \\
\hline MPV $4 \mathrm{~h}$ after surgery [fl] & $10.36 \pm 0.90$ \\
\hline PDW before surgery [fl] & $12.54 \pm 1.78$ \\
\hline PDW $4 \mathrm{~h}$ after surgery [fl] & $12.31 \pm 1.57$ \\
\hline \multicolumn{2}{|l|}{ C-reactive protein } \\
\hline Before surgery $[\mathrm{mg} / \mathrm{l}]$ & $10.38 \pm 14.63$ \\
\hline $72 \mathrm{~h}$ after surgery [mg/l] & $214.95 \pm 63.68^{\star}$ \\
\hline
\end{tabular}

Values are expressed as numbers or means $\pm S D,{ }^{*} p<0.05$ vs. prior to surgery 
Table II. Operative details

\begin{tabular}{|lc|}
\hline Operative time [min] & $121.79 \pm 36.30$ \\
\hline Aortic cross-clamping time [min] & $35.71 \pm 11.91$ \\
\hline Blood loss during operation [ml] & $928.57 \pm 849.82$ \\
\hline $\begin{array}{l}\text { Intravenous fluid supplementation } \\
\text { during surgery [ml] }\end{array}$ & $3496.43 \pm 1018.76$ \\
\hline $\begin{array}{l}\text { Erythrocyte mass transfusion during } \\
\text { surgery [ml] }\end{array}$ & $1120 ; 560$ \\
\hline Plasma transfusion during surgery [ml] & 1020 \\
\hline
\end{tabular}

Data in brackets specify the number of patients. Values are expressed as means $\pm S D$ or amounts

\pm standard deviation (mean \pm SD). Statistical significance was set at $p<0.05$.

\section{Results}

There were no differences between baseline and postoperative blood morphology parameters, except platelet counts (Table I), indicating that blood loss and supplementation were balanced during surgery, and, consequently, the blood supply to the kidneys was satisfactory. After surgery, serum creatinine rose (Table III), though not significantly. Preoperative eGFRs calculated according to MDRD Study and CKD-EPI Study equations were 74.39 $\pm 25.65 \mathrm{ml} / \mathrm{min} / 1.73 \mathrm{~m}^{2}$ and $88.84 \pm 23.47 \mathrm{ml} /$ $\mathrm{min} / 1.73 \mathrm{~m}^{2}$, respectively. Serum ICAM-1, VCAM-1, P-selectin, E-selectin and plasma vWF levels were compared as follows: 1) between samples taken before surgery from a peripheral vein (a) and all samples taken from different blood vessels at successive time points $(b-l), 2$ ) between samples taken from the renal vein at the start of surgery, i.e., before aortic clamping (b) and samples obtained from the renal vein, vena cava inferior, and peripheral veins just before aortic clamp release $(c, d, e)$ as well as those obtained from the renal and peripheral veins 5 min after clamp release $(f, g)$, 3) between samples taken from the renal vein just before aortic clamp release (c) and samples taken from the vena cava inferior and peripheral veins also before clamp removal (d, e) and, additionally, samples obtained $5 \mathrm{~min}$ after clamp release from renal and peripheral veins ( $f, g), 4)$ between samples obtained from the vena cava inferior (d) and peripheral vein (e) just before aortic clamp release, 5) between samples taken from the renal vein (f) and peripheral vein (g) 5 min after clamp release.
The only significant concentration changes were those in VCAM-1 levels: A) a decrease in all blood samples drawn during surgery just before and after aortic clamp release and an increase at $24 \mathrm{~h}$ and $48 \mathrm{~h}$ after clamp release as compared to day -1 (baseline), B) a decrease in all blood samples drawn during surgery just before and 5 min after aortic clamp release compared to renal vein levels before aortic clamping (baseline), C) a decrease in blood samples obtained from the renal vein 5 min after clamp release compared to renal vein levels just before clamp release (baseline) (Table IV).

The changes in ICAM-1 and P-selectin (Table IV) were similar to the VCAM-1 pattern, particularly in blood samples taken during surgery, but did not reach the level of statistical significance. E-selectin levels remained unchanged. vWF rose non-significantly in almost all samples taken from different blood vessels at successive time points as compared to day -1 (baseline). We also correlated serum creatinine with all serum SCAMs and vWF levels in the same samples on days (-1), (1) and (2). A significant correlation $(p<0.05)$ between serum creatinine and ICAM-1 was only found on day -1 ( $r s=0.6271)$. Time of aortic clamping was correlated with all serum sCAMs, creatinine and VWF; the only significant correlation was that with $\mathrm{P}$-selectin $2 \mathrm{~h}$ after clamp release ( $r s=0.5790)$. As P-selectin is principally released by activated platelets, we correlated the platelet count $(\mathrm{Plt})$ and parameters of platelet activation, i.e., platelet larger cell ratio (P-LCR), mean platelet volume (MPV) and platelet distribution width (PDW) [20] in the blood drawn before surgery $($ day -1$)$ with sP-selectin in the same sample. At this time point, sP-selectin correlated significantly with P-LCR ( $r s=0.6088)$. We subsequently correlated the same platelet parameters determined in the blood sample drawn $4 \mathrm{~h}$ after surgery with $\mathrm{SP}$ selectin levels in all blood samples drawn during and after surgery. The following correlations proved significant: a) between MPV and sP-selectin as well as PDW and SP-selectin in blood samples drawn $4 \mathrm{~h}$ after clamp release ( $\mathrm{rs}=0.6872$ and $\mathrm{rs}=0.6315$, respectively); $b$ ) between $\mathrm{Plt}$ and $\mathrm{SP}$-selectin in blood samples drawn $24 \mathrm{~h}$ (day 1) following clamp release ( $r s=-0.6967)$.

\section{Discussion}

It is not easy to define factors responsible for kidney damage associated with AAA surgery. The aorta is cross-clamped below the renal arteries.

Table III. Serum creatinine levels on successive observation days

\begin{tabular}{|lcccccc|}
\hline Parameter & Day -1 & Day 1 & Day 2 & Day 3 & Day 4 & Day 5 \\
\hline Mean \pm SD $[\mathrm{mg} / \mathrm{dl}]$ & $1.10 \pm 0.29$ & $1.33 \pm 0.47$ & $1.34 \pm 0.66$ & $1.31 \pm 0.87$ & $1.28 \pm 0.65$ & $1.33 \pm 1.05$ \\
\hline Value of $p$ vs. day -1 & & 0.127 & 0.223 & 0.393 & 0.339 & 0.454 \\
\hline
\end{tabular}


Thus, blood flow to the kidneys should not be compromised. However, AKI could also develop in the absence of overt kidney ischemia [21]. The pathogenesis of AKI is known to be a complex and multifactorial phenomenon. Surgery of AAA could trigger heart dysfunction which results in decreased effective intravascular volume and consequently may lead to reduced renal blood flow [22]. The malfunction of endothelial cells of kidney microcirculation appears to play a pivotal role. The set of activated cytokines is responsible for vasoconstriction and inflammation. The activation of the coagulation system also contributes to the local ischemia, particularly of the kidney outer medulla. The outcome of the processes mentioned above is injury of the renal tubular epithelium [23].

The mean level of serum creatinine in our study population rose. Although insignificant (Table III; $p$ values between 0.127155 and 0.453747 ), the rise did indicate a tendency to the development of AKI.

The left renal vein was the only one accessible during surgery. Thus, blood sampled from this vein was assumed to represent the condition of both kidneys. For this reason all patients enrolled underwent (a) Doppler examination of both renal arteries to rule out renal artery stenosis, (b) ultrasound determination of long axes of the right and left kidneys to exclude any possible differences, and (c) ultrasound investigations of the urinary tract to exclude obstruction. No significant differences were found in the study population.

Abdominal aortic aneurysm surgery induces a systemic inflammatory response (SIR) $[9,10]$. Inflammatory status along with toxic metabolic waste products inflow to the vena cava inferior from ischemic lower extremities as well as oxidative stress associated with surgical trauma might contribute to AKI development following AAA surgery.

An approximately 20-fold increase in CRP levels observed on day 3 after surgery confirmed an ongoing inflammatory state. A more subtle insight into the pathophysiological processes after AAA surgery was provided by the five indicators selected for the study, i.e., ICAM-1, VCAM-1, P-selectin, E-selectin and VWF. The first three showed a similar change pattern. Compared to baseline values, their levels at first decreased and after several hours increased again, the magnitude of these changes being different for each of the markers. P-selectin and ICAM-1 elevations were quite slight while the changes in VCAM-1 levels were significant. The E-selectin concentrations remained unchanged throughout the study. Plasma vWF, referred to as the "gold standard" for assessment of endothelial injury, demonstrated the expected, although non-significant, changes in the form of a slight intraoperative and a more marked postoperative increase. The first dilemma is associated with the initial fall of ICAM-1,

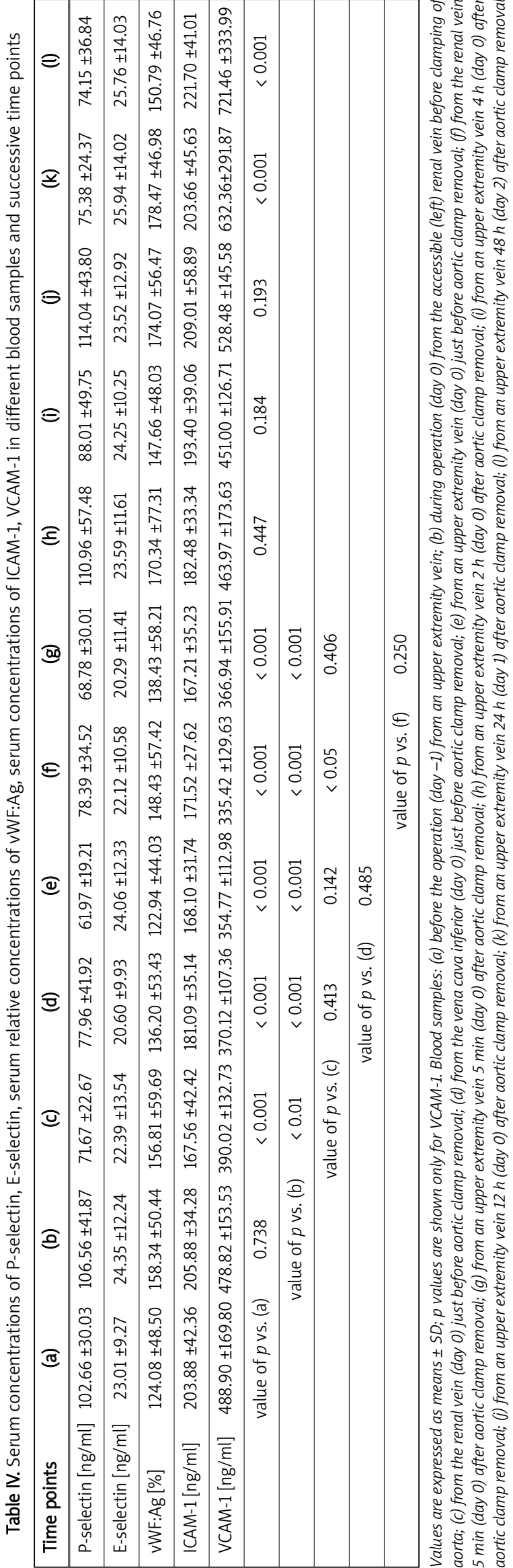


VCAM-1 and P-selectin concentrations. This biphasic response was quite unexpected and difficult to account for.

In humans, CAMs basically occur in two different forms: as membrane (mCAMs) and soluble cell adhesion molecules (sCAMs) [24]. In our study we determined the levels of sCAMs, i.e., sP-selectin, sE-selectin, sICAM-1, and SVCAM-1, but not of mCAMs. sCAMs only provide indirect insight into the function of membrane-bound forms. There are data showing parallelism of expression of cell surface-bound and soluble forms. The peak expression of sP-selectin during acute coronary syndromes only has a 2-hour delay in relation to its peak expression on platelets [25-27]. But another experiment has shown that, following aorta surgery, serum levels of SVCAM-1 and sICAM-1 might remain unchanged despite a significant increase of their tissue counterparts in kidneys [9]. This indicates that the relationship between membrane-bound and soluble forms might be quite complex. The origin and the function of SCAMs have not been well recognized so far; they appear in the plasma and other body fluids due to direct "secretion" from the cell, shedding or active cleavage from the cell surface [28]. The physiological/pathophysiological function of soluble forms is even more vague than their origin. They could competitively bind to ligands and hence block the function of their cell surface-bound counterparts. Therefore, the soluble type could attenuate cellular cooperation dependent on ligand-receptor interaction. However, after binding to a receptor, it could also activate different signaling pathways and, in consequence, modify cell functions [29]. Hence, although its role in the modulation of cellular interactions seems important, it is still poorly understood.

As mentioned above, the initial CAMs response to injury (i.e. AAA surgery) was quick but paradoxical; instead of the expected increase, their levels were found to have decreased. Although not easy, it is still possible to account for the phenomenon. Physiologically, a balance exists between the production and clearance of the soluble form. There is also a relatively constant cell surface density of both the membrane-bound form and its receptor. The density of ligands and their receptors should increase on cell surfaces following the activation of inflammatory processes. During the first phase, i.e., before the CAMs-dependent cell adhesion fully develops, the soluble and very mobile form (ligand) could easily saturate the strongly up-regulated receptors, and, in consequence, the levels of sCAMs decrease. Another consequence is temporary inability of mCAMs to bind to the already "engaged" receptors. Thus, the inflammatory process is momentarily suppressed. Then, in the second phase, the now increasingly up-regulated membrane form (ligand): a) is intensively shed to plasma, and b) combines with the remaining free receptors, saturating them and generating cellular adhesions. As a result of a) and b) the concentration of the soluble form increases. In our study three of four CAMs more or less distinctly showed the same biphasic pattern, which enhances the probability of the above hypothesis. Our general impression is that, particularly during the first phase, sCAMs could have modulated (suppressed) the inflammatory process during AAA surgery.

The complete non-reactivity of sE-selectin came as another surprise, and mainly because the primary injury in our study was to the biggest blood vessel in the body, i.e., the aorta. E-selectin is only expressed on EC and its level should selectively represent endothelial function [30]. A significant (even 4.5-fold) elevation of sE-selectin has been observed during coronary events, e.g., myocardial infarction [31, 32]. Thus, the complete unresponsiveness of E-selectin observed in our study remains to be elucidated.

Yet another problem deserves more attention. For a long time now VWF has been considered the gold standard marker of endothelial damage $[13,14]$. In our study, however, it reacted sluggishly, with nonsignificant increases. A slight increase was seen in renal vein samples at the beginning of surgery (Table IV, sample "b"), whereas the highest increases occurred at 2, 12 and $24 \mathrm{~h}$ after clamp release. Unlike P-selectin, ICAM-1 and VCAM-1, VWF demonstrated one-phase but erratic increase. It also contrasted with E-selectin, which, as already mentioned, showed no changes at all. An animal model of Sutton et al. [33] showed significant elevation of circulating VWF $24 \mathrm{~h}$ after ischemia-induced AKI. The actin cytoskeleton and adherens junctions of renal microvascular endothelium were disrupted. In another animal model of cisplatin-induced acute renal failure, the systemic VWF peaked 2 days after administration of cisplatin, which coincided with maximum expression of fractalkine - a potent chemoattractant and adhesion molecule in kidney endothelial cells [34]. In our model, a direct insight into EC submicroscopic changes was not possible, but we were able to assess changes in vWF levels in the renal vein, which constitutes a strength of the study. However, a doubt remains as to whether vWF elevation in the renal vein before aortic clamping (b) is a very early sign of renal endothelial injury or represents additional production of the factor by the kidney. The fact of disappearance of differences between renal ( $f$ ) and peripheral (g) veins 5 min after clamp release weighs in favor of the first option, which is also consistent with the fact that VWF may be released within minutes of insult; its increases can be evoked by minute stimuli or injuries such as vascular catheterization [35]. The biggest, although still insignificant, increases of vWF, which occurred at 2 (h), 12 (j) and 24 (k) h after clamp release, might 
be a summary expression of renal and systemic endothelial dysfunction. In dogs after endotoxin challenge, vWF level increased progressively to 2.1 times prechallenge values in $4 \mathrm{~h}$ [36]. vWF levels peaked significantly $4 \mathrm{~h}$ to $6 \mathrm{~h}$ after endotoxin administration to eleven normal volunteers, and remained elevated for $48 \mathrm{~h}$ [37]. In contrast to these results, the VWF increase noted in our patients was relatively weak and non-significant. This fact could, to some extent, harmonize with non-responsiveness of E-selectin. Despite being considered a useful indicator of endothelial dysfunction, the expression of VWF cannot be regarded as endothelium-specific. In contrast to E-selectin, vWF is also synthesized in megakaryocytes, stored in $\alpha$-granules of platelets and released during their activation. Most of the circulating VWF should derive from EC; however, in certain disorders, platelets might constitute a more efficient source of vWF than EC. vWF is also an acute phase reactant $[13,38]$. Since AKI investigated in our study is an inflammatory process, vWF might have increased as a marker of EC dysfunction or an acute phase reactant, or might have represented both conditions. Considering the changing levels of platelet activation marker (P-selectin) and unchanged values of EC-specific E-selectin, a question arises as to what extent VWF represented EC injury and not platelet activation.

The limitation of our study is the small sample size. However, the present report only describes the results of a pilot study. The strength of this study and its novel aspect is the possibility to assess the levels of inflammation/EC injury markers directly in the renal vein during AAA surgery. This allows a more direct insight into the events taking place in the kidney during AAA surgery.

We conclude that, during AAA surgery, P-selectin, ICAM-1 and VCAM-1 demonstrate a biphasic response with an initial fall and subsequent rise. The strongest reaction was characteristic of VCAM-1, which is an indicator not only of endothelial injury, but also of general inflammatory status. The soluble forms of cellular adhesion molecules might exert some modulatory effect on the inflammatory process. The unexpected non-reactivity of E-selectin along with a relatively weak VWF increase suggests that endothelial injury associated with AAA surgery is rather confined.

\section{References}

1. Lassnigg A, Schmidlin D, Mouhieddine M, et al. Minimal changes of serum creatinine predict prognosis in patients after cardiothoracic surgery: a prospective cohort study. J Am Soc Nephrol 2004; 15: 1597-605.

2. Thakar CV, Worley S, Arrigain S, Yared JP, Paganini EP. Influence of renal dysfunction on mortality after cardiac surgery: modifying effect of preoperative renal function. Kidney Int 2005; 67: 1112-9.
3. Rosner MH, Okusa MD. Acute kidney injury associated with cardiac surgery. Clin J Am Soc Nephrol 2006; 1: 19-32.

4. Ranucci M, Soro G, Barzaghi N, et al. Fenoldopam prophylaxis of postoperative acute renal failure in high-risk cardiac surgery patients. Ann Thorac Surg 2004; 78: 1332-7.

5. Hagivara S, Saima S, Negishi K, et al. High incidence of renal failure in patients with aortic aneurysms. Nephrol Dial Transplant 2007; 22: 1361-8.

6. Basile DP. The endothelial cell in ischemic acute kidney injury: implications for acute and chronic function. Kidney Int 2007; 72: 151-6.

7. Kinsey GR, Li L, Okusa MD. Inflammation in acute kidney injury. Nephron Exp Nephrol 2008; 109: e102-7.

8. Bonventre JV, Zuk A. Ischemic acute renal failure: an inflammatory disease? Kidney Int 2004; 66: 480-5.

9. Lozano FS, Rodriguez JM, Garcia-Criado FJ, et al. Postoperative evolution of inflammatory response in a model of suprarenal aortic cross-clamping with and without hemorrhagic shock. Systemic and Local Reactions. World J Surg 2005; 29: 1248-58.

10. Nishiyama T. Antithrombin can modulate coagulation, cytokine production, and expression of adhesion molecules in abdominal aortic aneurysm repair surgery. Anesth Analg 2006; 102: 1007-11.

11. Sumpio BE, Riley JT, Dardik A. Cells in focus: endothelial cell. Int J Biochem Cell Biol 2002; 34: 1508-12.

12. Molitoris BA, Sutton TA. Endothelial injury and dysfunction: role In the extension phase of acute renal failure. Kidney Int 2004; 66: 496-9.

13. Blann AD. Plasma von Willebrand factor, thrombosis, and the endothelium: the first 30 years. Thromb Haemost 2006; 95: 49-55.

14. Constans J, Conri C. Circulating markers of endothelial function in cardiovascular disease. Clin Chim Acta 2006; 368: 33-47.

15. Zamarrón C, Riveiro A, Gude F. Circulating levels of vascular endothelial markers in obstructive sleep apnoea syndrome. Effects of nasal continuous positive airway pressure. Arch Med Sci 2011; 7: 1023-8.

16. Tang IY, Murray PT. Prevention of perioperative ARF: what works? Best Pract Res Clin Anaesthesiol 2004; 18: 91-111.

17. Malyszko J. Biomarkers of acute kidney injury in different clinical settings: a time to change the paradigm? Kidney Blood Press Res 2010; 33: 368-82.

18. Delanaye P, Cavalier E, Mariat C, Maillard C, Krzesinski JM. MDRD or CKD-EPI study equations for estimating prevalence of stage 3 CKD in epidemiological studies: which difference? Is this difference relevant? BMC Nephrol 2010; 11: 8-14.

19. Malyszko J, Bachorzewska-Gajewska H, Malyszko J, lainaLevin N, Kobus G, Dobrzycki S. Markers of kidney function in the elderly in relation to the new CKD-EPI formula for estimation of glomerular filtration rate. Arch Med Sci 2011; 7: 658-64.

20. Polek A, Sobiczewski W, Matowicka-Karna J. P-selectin and its role in some diseases. Postepy Hig Med Dosw 2009; 63: 465-70.

21. Chang CK, Chuter TAM, Niemann CU, et al. Systemic inflammation, coagulopathy, and acute renal insufficiency following endovascular thoracoabdominal aortic repair. J Vasc Surg 2009; 49: 1140-6.

22. Waliszek M, Waliszek-Iwanicka A, Grycewicz T, et al. Prognostic value of plasma $\mathrm{N}$-terminal pro-B-type natriuretic peptide concentration in patients with normal and impaired left ventricular systolic function undergoing 
surgery for abdominal aortic aneurysm. Arch Med Sci 2011; 7: 642-7.

23. Bonventre JV, Yang L Cellular pathophysiology of ischemic acute kidney injury. I Clin Invest 2011; 121: 4210-21.

24. Mantur M, Wojszel J. Cell adhesion molecules and their participation in the process of inflammation and cancerogenesis. Pol Merkur Lekarski 2008; 24: 177-8.

25. Dunlop LC, Skinner MP, Bendal LJ, et al. Characterization of GMP-140 (P-selectin) as a circulating plasma protein. J Exp Med 1992; 174: 1147-50.

26. Michelson AD, Barnard MR, Hechtman HB, et al. In vivo tracking of platelets: circulating degranulated platelets rapidly loss surface P-selectin but continue to circulate and function. Proc Natl Acad Sci USA 1996; 93: 11877-82.

27. Shebuski RJ, Kilgore KS. Role of inflammatory mediators in thrombogenesis. J Pharmacol Exp Ther 2002; 300: 729-35.

28. Blann AD, Nadar SK, Lip GYH. The adhesion molecule P-selectin and cardiovascular disease. Eur Heart J 2003; 24: 2166-79.

29. Lawson C, Wolf S. ICAM-1 signaling in endothelial cells. Pharmacol Rep 2009; 61: 22-32.

30. Constans J, Conri C. Circulating markers of endothelial function in cardiovascular disease. Clin Chim Acta 2006; 368: 33-47.

31. Mashru MR, Shah VK, Soneji SL, et al. Soluble levels of cell adhesion molecules (CAMs) in coronary artery disease. Indian Heart J 2010; 62: 57-63.

32. Pellegatta F, Pizzetti G, Lu Y, et al. Soluble E-selectin and intercellular adhesion molecule-1 plasma levels increase during acute myocardial infarction. J Cardiovasc Pharmacol 1997; 30: 455-60

33. Sutton TA, Mang HE, Campos SB, Sandoval RM, Yoder MC, Molitoris BA. Injury of the renal microvascular endothelium alters barrier function after ischemia. Am J Physiol Renal Physiol 2003; 285: F191-8.

34. Lu LH, Oh DJ, Dursun B, et al. Increased macrophage infiltration and fractalkine expression in cisplatin-induced acute renal failure in mice. J Pharmacol Exp Ther 2008; 324: 111-7.

35. Newsholme SJ, Thudium DT, Gossett KA, Watson ES, Schwartz LW. Evaluation of plasma von Willebrand factor as a biomarker for acute arterial damage in rats. Toxicol Pathol 2000; 28: 688-93.

36. Novotny MJ, Turrentine MA, Johnson GS, Adams HR. Experimental endotoxemia increases plasma von Willebrand factor antigen concentrations in dogs with and without free-radical scavenger therapy. Circ Shock 1987; 23: 205-13.

37. Gralnick HR, McKeown LP, Wilson OM, Williams SB, Elin RJ. von Willebrand factor release induced by endotoxin. J Lab Clin Med 1989; 113: 118-22.

38. Lip GY, Blann A. von Willebrand factor: a marker of endothelial dysfunction in vascular disorders? Cardiovasc Res 1997; 34: 255-65. 